Structural Equation Modeling (SEM)-Based Meta-Analysis

Mike W.-L. Cheung ${ }^{1}$

${ }^{1}$ Department of Psychology, National University of Singapore

Author Note

Correspondence concerning this article should be addressed to Mike W.-L. Cheung, Department of Psychology, Faculty of Arts and Social Sciences, National University of Singapore, Block AS4, Level 2, 9 Arts Link, Singapore 117570. Tel: (65) 6516-3702; E-mail: mikewlcheung@nus.edu.sg.

Note. This is a pre-print of an invited chapter that will appear in Hoyle R. H. (Ed.) Handbook of Structural Equation Modeling (2nd edition). 


\begin{abstract}
Structural equation modeling (SEM) and meta-analysis are two popular techniques in the behavioral, medical, and social sciences. They have their own research communities, terminologies, models, software packages, and even journals. This chapter introduces SEMbased meta-analysis, an approach to conduct meta-analyses using the SEM framework. By conceptualizing studies in a meta-analysis as subjects in a structural equation model, univariate, multivariate, and three-level meta-analyses can be fitted as structural equation models using definition variables. We will review fixed-, random-, and mixed-effects models using the SEM framework. Examples will be used to illustrate the procedures using the metaSEM and OpenMx packages in R. This chapter closes with a discussion of some future directions for research.
\end{abstract}

Keywords: meta-analysis, structural equation modeling, SEM-based meta-analysis, fixedeffect model, random-effects model, mixed-effects model 


\section{Structural Equation Modeling (SEM)-Based Meta-Analysis}

Structural equation modeling (SEM) is an essential statistical modeling framework in the social and behavioral sciences. It provides a flexible framework for testing complicated models involving latent and observed variables (e.g., Bentler, 1986; Bollen, 1989; Jöreskog, 1967). SEM combines ideas of latent variables from psychometrics, path models from sociology, and structural models from econometrics. After several decades of development, SEM has integrated many statistical techniques into a single framework, such as the analysis of ordinal variables (Muthén, 1984), analysis of missing data (Enders, 2010), multilevel models (Muthén, 1994), and mixture models (Lubke \& Muthén, 2007), among others. The general linear model, path analysis, and confirmatory factor analysis are some special cases of SEM. Multilevel models (e.g., Bauer, 2003; Curran, 2003; Mehta \& Neale, 2005; Rovine \& Molenaar, 2000) and some item response theory models (e.g., Glockner-Rist \& Hoijtink, 2003; Takane \& Deleeuw, 1987) have also been shown to be special cases of SEM.

Meta-analysis is now the de facto method for use in synthesizing research findings in the social, behavioral, and medical sciences. Glass (1976) coined the term meta-analysis for “the statistical analysis of a large collection of analysis results” (p. 3). Hedges and Olkin (1985) provided the statistical foundation of meta-analysis. Schmidt and Hunter (2015) presented an alternative approach to conducting a meta-analysis, which is called validity generalization in industrial and organizational psychology and management. Borenstein et al. (2009) gave a comprehensive overview of statistical methods in meta-analysis. Using a systematic review and meta-analysis, researchers might be able to answer several crucial questions in research, such as (1) Are the research findings in a particular topic homogeneous (consistent)? (2) What is the best estimate of the average effect and its heterogeneity variance? (3) Can some moderators explain the heterogeneity variance? 
SEM and meta-analysis are usually treated as two separate topics in textbooks (e.g., Borenstein et al., 2009; Kline, 2016), software packages (e.g., Rosseel, 2012; Viechtbauer, 2010), graduating training courses (e.g., Aiken et al., 2008), and even journals (Structural Equation Modeling: A Multidisciplinary Journal and Research Synthesis Methods).

Researchers with knowledge in one area might not be aware of the benefits, techniques, and issues surrounding knowledge in other areas. Integrating meta-analysis into the SEM framework may open up many research avenues for developing new statistical methods and applications.

This chapter provides a brief introduction to the recent development of SEM-based meta-analysis (Cheung, 2008; 2010; 2013b; 2014a; 2018; 2019a), in which the SEM framework is used to conduct meta-analyses. Readers may refer to Cheung (2015a) for a book-length treatment of this topic. SEM provides a flexible framework to model metaanalytic data. There are several benefits to using the SEM approach to conduct a metaanalysis. First, linear and non-linear constraints on meta-analytic models can be implemented in some SEM packages, such as OpenMx and Mplus. Second, advanced data analytic methods, such as the analysis of non-normal data, missing data, parametric and nonparametric bootstrap, likelihood-based confidence intervals, and mixture models, are available in some SEM packages.

Table 1 provides a conceptual map of some key concepts in these fields. The essential idea in conceptualizing a meta-analysis as a structural equation model is to treat studies in a meta-analysis as subjects in a structural equation model. For example, if there are 50 studies in a meta-analysis, it means that there are 50 subjects in a structural equation model. The observed and population (or true) effect sizes in the meta-analysis can be treated as the observed and latent variables in SEM, respectively. The mean and variance of the effect size 
are called the average effect and heterogeneity variance in meta-analysis, respectively.

Each effect size has its own sampling variance (the inverse of the precision), mainly due to the difference in sample sizes. The trick to handling a meta-analysis as a structural equation model is to treat this known sampling variance as something similar to the measurement error in SEM. When there is an excess of heterogeneity, researchers may use some study characteristics to predict the effect sizes. In a meta-analysis, these study characteristics are sometimes called predictors, covariates, or moderators. They are called moderators because they moderate the magnitude of the effect. To avoid confusion, we use the term covariate in this chapter.

In the next section, we first review how to compute effect sizes and their sample variances, which are the building blocks of a meta-analysis. After that, we introduce a univariate meta-analysis under fixed-effect, random-effects, and mixed-effects models. We then present the extensions to a multivariate and three-level meta-analysis. We illustrate these analyses with two published datasets in the meta-analysis using the metaSEM (Cheung, 2015b) and OpenMx (Neale et al., 2016) packages implemented in R, an open-source statistical environment (R Development Core Team, 2021).

\section{Computing Effect Sizes}

Effect sizes are the primary data in a meta-analysis. Kelley and Preacher (2012) defined an effect size as " $a$ quantitative reflection of the magnitude of some phenomenon that is used for the purpose of addressing a question of interest” (p. 140, emphasis in original). Effect sizes can be either standardized or unstandardized. Table 2 shows some common effect sizes in a meta-analysis. To fix the notation, we use $y_{i}$ to represent a generic sample effect size in the ith study,

$$
y_{i}=f_{i}+e_{i},
$$


where $f_{i}$ is the population (or true) effect size, and $\operatorname{Var}\left(e_{i}\right)=v_{i}$ is its sampling variance, which in a meta-analysis is usually assumed to be known. $y_{i}$ can be a standardized mean difference, correlation coefficient, or other effect size, depending on the research questions. Once we have calculated the effect sizes and their sampling variances, the same meta-analytic procedure can be applied regardless of the type of effect size.

A few points should be noted in choosing appropriate effect sizes. First, the chosen effect sizes should provide directions on the effect. Effect sizes associated with the percentage of variances explained, such as $R^{2}, \eta^{2}$, and $\Omega^{2}$, are rarely used in a meta-analysis. Second, some estimators have a small sample bias. For example, the standardized mean difference (SMD) in Table 2 defined by the difference between two group means divided by the pooled standard deviation is slightly biased in a small sample. A small correction factor may be applied to the effect sizes to minimize the bias (Hedges, 1981). Third, the effect size is assumed to be distributed normally with the known sampling variance. That is, $y_{i} \sim N\left(f_{i}, v_{i}\right)$. Most effect sizes only approach a normal distribution asymptotically. Thus, a reasonable sample size is required in the primary studies.

Fourth, the sampling distributions of some estimators approach a normal distribution faster than others do. Researchers may apply a variance stabilizing transformation on the effect size. For example, a log transformation is always applied to the relative risk (RR) and odds ratio (OR). After the meta-analysis, an inverse transformation may apply to facilitate the interpretations. The case of the correlation coefficient is slightly more complicated. A Fisher's $z$ transformation is sometimes applied to the correlation coefficient to make its sampling distribution more quickly approach a normal distribution. In some fields, such as industrial and organizational psychology and management, researchers prefer to use the correlation coefficient because statistical and measurement artifacts on the correction can be corrected 
before the meta-analysis is conducted (Schmidt \& Hunter, 2015).

Since many effect sizes are functions of the means and covariance matrices, SEM can be used to compute the effect sizes. Their sampling variances and covariances can be calculated via the Delta method implemented in many SEM packages (Cheung, 2015a, Chapter 3; Raykov \& Marcoulides, 2004). The SEM approach is helpful for calculating effect sizes in complex designs, such as the multiple-treatment studies and multiple-endpoint studies common in experimental design involving several treatment groups or outcome variables (Cheung, 2018). Since the maximum likelihood (ML) estimation method is used in most SEM packages, the SEM approach to computing the effect sizes and their sampling variances works well only when the sample sizes are reasonably large.

\section{Univariate Meta-Analysis}

In this section, we introduce various models for conducting a meta-analysis with one type of effect. These models include the fixed-effect, random-effects, and mixed-effects models.

\section{Fixed-Effect Model}

The simplest meta-analytic model is a fixed-effect model, also known as the commoneffect model,

$$
y_{i}=\beta_{\mathrm{F}}+e_{i},
$$

where $\beta_{\mathrm{F}}=f_{1}=f_{2}=\ldots=f_{k}$ is the common population effect for $k$ studies. The unknown parameter and its sampling variance can be estimated with the weighted least squares (WLS),

$$
\begin{gathered}
\hat{\beta}_{\mathrm{F}}=\frac{\sum_{i=1}^{k} w_{i} y_{i}}{\sum_{i=1}^{k} w_{i}}, \\
\operatorname{Var}\left(\hat{\beta}_{\mathrm{F}}\right)=\frac{1}{\sum_{i=1}^{k} w_{i}}, \\
\operatorname{SE}\left(\hat{\beta}_{\mathrm{F}}\right)=\sqrt{\operatorname{Var}\left(\hat{\beta}_{\mathrm{F}}\right)},
\end{gathered}
$$


where $w_{i}=1 / v_{i}$. The estimator in Equation (3) is an unbiased estimate of the common population effect if $y_{i}$ is unbiased and the population effect sizes are homogeneous. Moreover, it has the smallest sampling variance among all possible weighted estimators in the class of unbiased estimators when the sampling variances are truly known (Hedges, 2007). We may test the null hypothesis $H_{0}: \beta_{\mathrm{F}}=\beta_{0}$ by using

$$
z=\frac{\hat{\beta}_{\mathrm{F}}-\beta_{0}}{\operatorname{SE}\left(\hat{\beta}_{\mathrm{F}}\right)} .
$$

Under the null hypothesis, $z$ has an approximate standard normal distribution. We reject the null hypothesis at $\alpha=0.05$ when the absolute value of $z$ is equal to or larger than 1.96. Moreover, we can also construct a Wald CI to indicate the precision of the estimate.

Under the fixed-effect model, the differences in the observed effect sizes are assumed to be only due to the sampling error. Cochran (1954) proposed the following $Q$ statistic to test this hypothesis:

$$
Q=\sum_{i=1}^{k} w_{i}\left(y_{i}-\hat{\beta}_{\mathrm{F}}\right)^{2} .
$$

Under the null hypothesis of the homogeneity effect sizes, the $Q$ statistic has an approximate chi-square distribution with $(k-1)$ degrees of freedom $(d f s)$.

Figure 1 displays the fixed-effect model using the graphical representation in SEM. As we can see from the figure, the model is straightforward with only one unknown parameter $\beta_{\mathrm{F}}$. The model-implied mean and variance are

$$
\begin{aligned}
& \mu_{i}(\theta)=\beta_{\mathrm{F}}, \\
& \Sigma_{i}(\theta)=v_{i} .
\end{aligned}
$$

The $-2 * \log$-likelihood $\left(-2 L L_{i}\right)$ of the ith study is

$$
-2 L L_{i}(\theta)=k_{i} \log (2 \pi)+\log \left|\Sigma_{i}(\theta)\right|+\left(y_{i}-\mu_{i}(\theta)\right)^{\mathrm{T}} \Sigma_{i}(\theta)^{-1}\left(y_{i}-\mu_{i}(\theta)\right),
$$

where $k_{i}$ is the number of non-missing observed variables in the ith study, $|X|$ is the determinant of $X$, and $\log (x)$ is the natural logarithm of $x$ (e.g., Enders, 2010). As the 
studies are independent, the $-2 L L$ of all studies is the sum of $-2 L L_{i}$ over all studies. Parameter estimates and the sampling covariance matrix can be obtained using the full information maximum likelihood (FIML) estimation method implemented in the SEM software.

One challenge to analyzing this model is that the known sampling variance $v_{i}$ has to be fixed at each row (subject). However, most SEM packages, such as lavaan (Rosseel, 2012), EQS (Bentler, 2006), and LISREL (Jöreskog \& Sörbom, 1996), do not allow this. We need to use either the definition variable in OpenMx or the CONSTRAINT command in Mplus to impose the known sampling variance on $y_{i}$. Alternatively, we may apply a transformation to the model so that the known sampling variances become one (Cheung, 2008). The transformed model can be fitted with standard SEM packages.

The fixed-effect model assumes that the population effect sizes are the same. However, studies might differ in various ways, such as by design, measure, population, or cohort. The estimated standard errors (SEs) are under-estimated when the effect sizes are heterogeneous.

\section{Random-Effects Model}

A more realistic model is the random-effects model that allows heterogeneity in the population effect sizes,

$$
y_{i}=\beta_{\mathrm{R}}+u_{i}+e_{i},
$$

where $\beta_{\mathrm{R}}$ is the average effect under the random-effects model and $\operatorname{Var}\left(u_{i}\right)=\tau^{2}$ is the heterogeneity variance that has to be estimated. The parameters can be estimated via a twostep approach. In the first step, $\tau^{2}$ is estimated by a weighted or unweighted method of moments (e.g., DerSimonian \& Laird, 1986; Hedges, 1983). In the second step, the $\beta_{\mathrm{R}}$ and its sampling variance are estimated, 


$$
\begin{gathered}
\hat{\beta}_{\mathrm{R}}=\frac{\sum_{i=1}^{k} \tilde{w}_{i} y_{i}}{\sum_{i=1}^{k} \tilde{w}_{i}}, \\
\operatorname{Var}\left(\hat{\beta}_{\mathrm{R}}\right)=\frac{1}{\sum_{i=1}^{k} \tilde{w}_{i}},
\end{gathered}
$$

where $\tilde{w}_{i}=1 /\left(\hat{\tau}^{2}+v_{i}\right)$ is the new weight taking the heterogeneity variance into account. Apart from the method of moments, the ML estimation and restricted maximum likelihood (REML) estimation methods may also be used. Interested readers may refer to Viechtbauer (2005) for an empirical comparison of the performance of common estimators in metaanalysis.

Statistically, the fixed-effect model is a special case of the random-effects model when the heterogeneity variance is zero. These two models serve different conceptual purposes (Hedges \& Vevea, 1998). The fixed-effect model focuses on those studies in the current meta-analysis with the same design, measures, and samples. In contrast, the intention in a random-effects model is to generalize the findings beyond the studies included in the current meta-analysis. There are other models, such as a fixed-effect model without any assumptions made about the homogeneity of effect sizes (Bonett, 2008) and a neither fixed nor random model (Stanley \& Doucouliagos, 2015).

Although the $Q$ statistic in Equation (5) may be used to test the homogeneity of effect sizes, it does not quantify the degree of heterogeneity. An obvious choice is the estimated $\tau^{2}$, which indicates the variance in the scale of $y_{i}$. One potential limitation is that there is no transparency with regard to what a particular value for $\tau^{2}$, say 0.1 , might mean in a raw mean difference or a standardized mean difference. This makes it difficult to benchmark how large or small the heterogeneity is. Higgins and Thompson (2002) proposed several indices to quantify the heterogeneity of effect sizes that are independent of the types of effect sizes. The most popular one is the $I^{2}$ : 


$$
I^{2}=\frac{Q-(k-1)}{Q}
$$

When the calculated $I^{2}$ is negative, it is truncated to 0 . Another way to express $I^{2}$ is

$$
I^{2}=\frac{\hat{\tau}^{2}}{\hat{\tau}^{2}+\tilde{v}}
$$

where $\tilde{v}$ is a typical within-study sampling variance (Takkouche et al., 1999; Xiong et al., 2010). $I^{2}$ can be interpreted as the proportion of the total variation of the effect size due to the between-study variance, which is similar to the intra-class correlation (ICC) in a multilevel model.

Higgins et al. (2003) suggested that an $I^{2}$ of $25 \%, 50 \%$, and $75 \%$ can be regarded as low, moderate, and high heterogeneity, respectively. Several remarks are noteworthy. First, these cutoffs were based on meta-analyses published in medical research. It remains unclear how they can be applied to other fields. Second, $I^{2}$ approaches one when the sample sizes in the primary studies are getting larger and larger. Third, $I^{2}$ is a descriptive statistic, that depends on sample size. Thus, it is not estimating any population parameters.

Figure 2 displays a graphical model of the random-effects model. The central idea is to treat the population effect size as a latent variable. The model-implied mean and variance are

$$
\begin{gathered}
\mu_{i}(\theta)=\beta_{\mathrm{R}}, \\
\Sigma_{i}(\theta)=\tau^{2}+v_{i} .
\end{gathered}
$$

The FIML estimation implemented in the SEM software can be used to fit the model. By applying a transformation matrix, the REML estimation method (Cheung, 2013a) can also be implemented in the SEM software. Statistical inferences can be made based on the parameter estimates and their sampling covariance matrix. Once we have conceptualized the population effect sizes as latent variables, complicated models involving the population effect sizes as predictors or outcome variables can be formulated and tested easily under the SEM 
framework.

\section{Mixed-Effect Model}

The random-effects model assumes that the heterogeneity variance is due to a random process. Researchers sometimes want to know how the effects vary as a function of some covariates or study characteristics. When the covariates are categorical, we may run separate meta-analyses on each level, which is known in the literature as a subgroup analysis. The same analysis can be easily implemented in SEM by using a multiple-group analysis (Schoemann, 2016). A more general approach is to use the mixed-effects meta-analysis, also known as the meta-regression. The model with a covariate $x_{i}$ in the ith study is

$$
y_{i}=\beta_{0}+\beta_{1} x_{i}+u_{i}+e_{i},
$$

where $\beta_{0}$ is the intercept when $x_{i}$ is $0, \beta_{1}$ is the expected change when $x_{i}$ increases by one unit, and $\operatorname{Var}\left(u_{i}\right)=\tau^{2}$ is the residual heterogeneity variance after controlling for the covariate.

There are two approaches to fit the mixed-effects model depending on whether $x_{i}$ is treated as a variable or a design matrix (Cheung, 2015a). Figure 3 shows a model treating $x_{i}$ as a variable, which is common in SEM. We need to estimate the mean and variance of $x_{i}$ in this model,

$$
\begin{gathered}
\mu_{i}(\theta)=\mathrm{E}\left(\left[\begin{array}{l}
y_{i} \\
x_{i}
\end{array}\right]\right)=\left[\begin{array}{c}
\beta_{0}+\beta_{1} \mu_{x} \\
\mu_{x}
\end{array}\right], \\
\Sigma_{i}(\theta)=\operatorname{Cov}\left(\left[\begin{array}{c}
y_{i} \\
x_{i}
\end{array}\right]\right)=\left[\begin{array}{cc}
\beta_{1}^{2} \sigma_{x}^{2}+\tau^{2}+v_{i} & \\
\beta_{1} \sigma_{x}^{2} & \sigma_{x}^{2}
\end{array}\right] .
\end{gathered}
$$

There are two limitations to this model. First, studies with missing values in $x_{i}$ have to be dropped before the analysis. This may lead to problems because having incomplete study characteristics is a rule rather than an exception in meta-analyses. The second limitation is that a normal distribution has to be assumed when estimating the mean and variance of $x_{i}$. This assumption might not be appropriate, especially when there are binary or categorical covariates. 
In the literature on SEM, it is well known that FIML can be used to handle missing covariates by treating the covariates as dependent variables (e.g., Enders, 2010). To address the first concern of missing covariates, Cheung (2015a, Chapter 8) showed how we could handle missing covariates with the model in Figure 4 by treating the covariate as a dependent variable. Assuming that the missing covariates are missing at random (MAR), FIML can be used to fit the model without dropping any studies with missing values. However, we still need to assume that the covariates are normally distributed. This concern can be potentially addressed by the use of robust SE (Yuan et al., 2012), as robust SE with FIML have been found to work reasonably well on nonnormal incomplete data.

Another approach is to treat $x_{i}$ as a design matrix (fixed values), which is common in meta-analysis, regression analysis, and multilevel models. Figure 5 shows a model with a phantom variable $p$ with a variance of 0 (Rindskopf, 1984). The conditional model-implied mean and variance are

$$
\begin{gathered}
\mu_{i}\left(\theta \mid x_{i}\right)=x_{i} \beta, \\
\Sigma_{i}\left(\theta \mid x_{i}\right)=\tau^{2}+v_{i} .
\end{gathered}
$$

This model can be fitted with the use of definition variables. Since $x_{i}$ is not a variable, there is no distribution assumption on it. However, missing covariates have to be dropped before the analysis.

After fitting the mixed-effects meta-analysis, researchers may calculate an $R^{2}$ index to indicate the percentage of the variance that can be explained by the covariates (e.g., Borenstein et al., 2009; Raudenbush, 2009),

$$
R^{2}=\frac{\hat{\tau}_{0}^{2}-\hat{\tau}_{1}^{2}}{\hat{\tau}_{0}^{2}}
$$

where $\hat{\tau}_{1}^{2}$ and $\hat{\tau}_{0}^{2}$ are the estimated heterogeneity variances with and without the covariates, respectively. Two issues should be noted here. First, the estimated $R^{2}$ can be negative. When 
that happens, the estimated $R^{2}$ is truncated to zero. Second, if there are missing values in the covariates, these cases have to be excluded before the heterogeneity variances are calculated; otherwise, these two estimates will be based on different numbers of studies.

An alternative $R^{2}$, which is popular in the SEM literature, is the one based on a model with the covariates,

$$
\tilde{R}^{2}=\frac{\hat{\beta}_{1}^{2} \sigma_{x}^{2}}{\hat{\beta}_{1}^{2} \sigma_{x}^{2}+\hat{\tau}_{1}^{2}} .
$$

It offers several practical advantages to the one in Equation (16). First, it only requires fitting one model. Second, users do not need to worry about the differences in sample sizes as only one model is fitted. Third, this estimator is always non-negative. Since this estimator is rarely used in meta-analysis, future research might be needed to see how it compares to Equation (16).

\section{Multivariate Meta-Analysis}

So far, we have only focused on meta-analyses with one outcome effect size. The situations tend to be much more complicated in reality. For example, there are two effect sizes (math subtest and verbal subtest) in studying the effectiveness of coaching on performance on the Scholastic Aptitude Test (Raudenbush et al., 1988). It is expected that both the math and verbal subsets will be correlated. Another example is the meta-analysis of diagnostic tests (Dimou et al., 2016). The outcome effect sizes are the sensitivity and specificity of a test, both of which are correlated. A multivariate meta-analysis extends the univariate meta-analysis by analyzing several correlated effect sizes (e.g., Kalaian \& Raudenbush, 1996; Jackson et al., 2011; van Houwelingen et al., 2002).

Because not all studies report all effect sizes, we need some notations to handle incomplete effect sizes. Let $\boldsymbol{X}_{0}$ and $\boldsymbol{Z}$ be two selection matrices of 0 and 1 to filter the appropriate elements in the fixed effects and random effects, respectively. Since the fixed- 
effects model is a special case of the random-effects model, we will focus on the randomeffects model here:

$$
\boldsymbol{y}=\boldsymbol{X}_{0} \boldsymbol{\beta}_{\mathrm{R}}+\boldsymbol{Z} \boldsymbol{u}+\boldsymbol{e},
$$

where $\boldsymbol{y}$ is a column vector of all effect sizes stacked together, $\boldsymbol{\beta}_{\mathrm{R}}$ is a vector of the average effects, $\operatorname{Cov}\left(\boldsymbol{u}_{i}\right)=\mathbf{T}^{2}$ is the heterogeneity variance-covariance matrix that has to be estimated, and $\operatorname{Cov}\left(\boldsymbol{e}_{i}\right)=\boldsymbol{V}_{i}$ is the known sampling variance-covariance matrix in the $i$ th study.

It is easier to illustrate the idea with an example of two effect sizes. Suppose the first study includes two effect sizes $\mathbf{y}_{(1)}$, while the second and third studies ( $\mathbf{y}_{(2)}$ and $\left.\mathbf{y}_{(3)}\right)$ report only one effect size; the model is

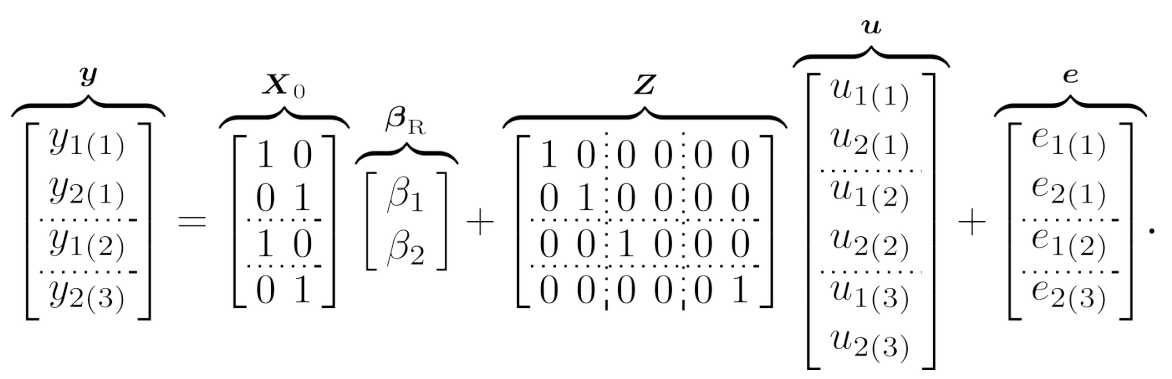

A few points should be noted here. First, the sampling errors are correlated within a study $\left(\boldsymbol{V}_{i}\right)$ because the same subjects are involved in calculating the effect sizes in the ith study. Second, the heterogeneity matrix $\left(\mathbf{T}^{2}\right)$, which is assumed to be positive definite, indicates the variances and covariance of the population effect sizes. When there are too many outcome effect sizes, there might not be sufficient data to estimate the full $\mathbf{T}^{2}$. One way of working around this problem is to assume $\mathbf{T}^{2}$ diagonals with the assumption that the population effect sizes are uncorrelated. Third, the estimators in the univariate meta-analysis can be extended to the multivariate meta-analysis (e.g., Jackson et al., 2011; Jackson et al., 2013). Fourth, we may calculate an $I^{2}$ index on each outcome effect size using Equations (10) or (11). An alternative is to extend the univariate $I^{2}$ to its multivariate counterpart (see Jackson et al., 2012). 
The formulation in Equation (18) is based on the long format of data under a multilevel model approach. Cheung (2013b) showed how the multivariate meta-analysis could be analyzed as a structural equation model using the wide format of data. Figure 6 shows a multivariate meta-analysis with two effect sizes. The model implied vector and covariance matrix are

$$
\begin{gathered}
\boldsymbol{\mu}_{i}(\boldsymbol{\theta})=\boldsymbol{\beta}_{\mathrm{R}}, \\
\boldsymbol{\Sigma}_{i}(\boldsymbol{\theta})=\boldsymbol{T}^{2}+\boldsymbol{V}_{i} .
\end{gathered}
$$

The known sampling covariance matrices are conceptualized as correlated measurement errors implemented via definition variables. The between-study heterogeneity covariance matrix $\left(\mathbf{T}^{2}\right)$ is regarded as the variance-covariance matrix of the latent factors. When there are incomplete effect sizes, the missing values are handled using the FIML estimation method, on the assumption that the missing data are missing at random (MAR).

Similar to the univariate meta-analysis, we may include covariates to explain the heterogeneity in the multivariate meta-analysis. The mixed-effects meta-analysis is

$$
\boldsymbol{y}=\boldsymbol{X}_{0} \boldsymbol{\beta}_{0}+\boldsymbol{X}_{1} \boldsymbol{\beta}_{1}+\boldsymbol{Z} \boldsymbol{u}+\boldsymbol{e},
$$

where $\boldsymbol{\beta}_{0}$ and $\boldsymbol{\beta}_{1}$ are vectors of the intercepts and slopes, respectively, $\boldsymbol{X}_{1}$ is a matrix of 0 (when the effect sizes are missing) and the values of the covariates (when the effect sizes are present). The random-effects model in the above example can be extended to a mixed-effects model with one covariate $x_{1}$, 

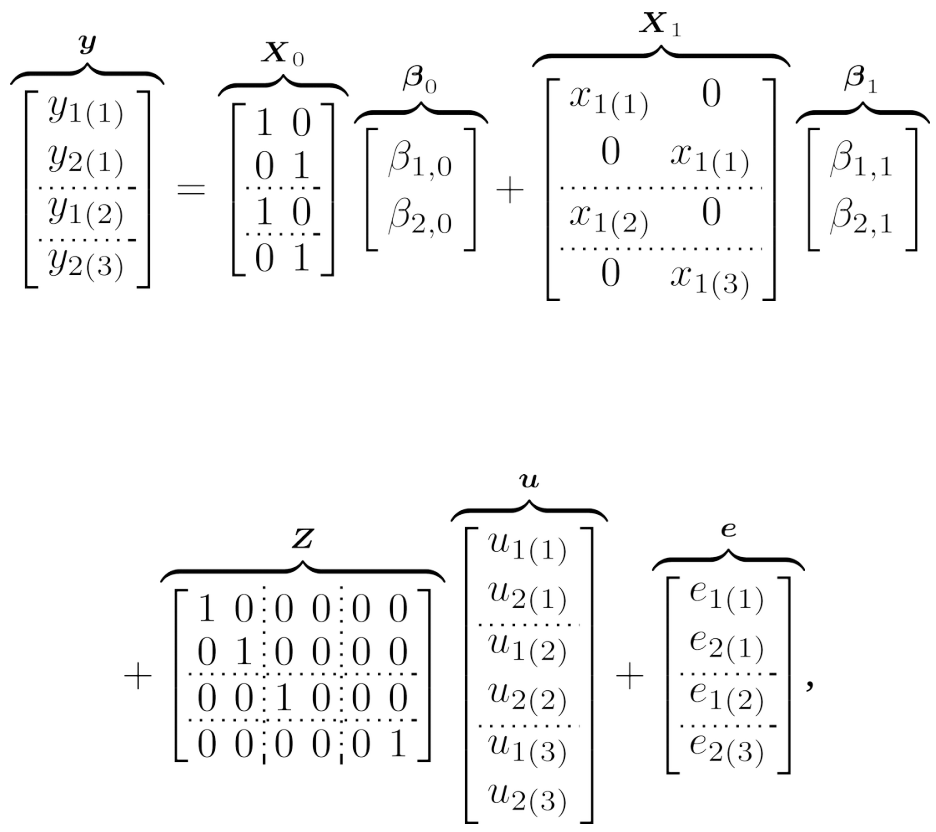

where $x_{1(2)}$ indicates the covariate $x_{1}$ in the second study. An $R^{2}$ index (Equation (16) can be calculated on each outcome effect size. Alternatively, we may also apply the multivariate version of $R^{2}$ proposed by Jackson et al. (2012).

Figure 7 shows the SEM approach to fitting a multivariate mixed-effects metaanalysis with two outcome effect sizes. The values of the covariates are fixed at the subject level by the use of definition variables. One main limitation of this model specification is that studies with missing covariates have to be dropped before the analysis. If it is reasonable to assume that the distribution of the covariates are multivariate normal and the missingness is MAR, the model in Figure 4 can be extended to handle incomplete covariates in a multivariate mixed-effects meta-analysis.

\section{Three-Level Meta-Analysis}

The multivariate model is useful for handling dependence in a meta-analysis. There is another type of dependence-effect sizes nested within clusters. For example, publications are nested within the same research teams or authors (e.g., Cooper, 2010). Effect sizes from the same research teams tend to be more similar than those from other teams. Another 
example is in a cross-cultural meta-analysis (e.g., Fischer \& Boer, 2011), in which effect sizes reported by studies are nested within countries. Participants from the same country or ethnic group may share similar values, beliefs, and psychological attributes. Thus, it is expected that published studies are more similar within countries than between countries.

A three-level meta-analysis (Konstantopoulos, 2011; Van den Noortgate et al., 2013) can be used to handle the extra level of dependence of the effect size $y_{i j}$ in the ith effect size in the $j$ th cluster:

$$
y_{i j}=\beta_{0}+u_{(2) i j}+u_{(3) j}+e_{i j},
$$

where $\beta_{0}$ is the average population effect, $\operatorname{Var}\left(u_{(2) i j}\right)=\tau^{2}{ }_{(2)}$ is the level-2 heterogeneity variance, $\operatorname{Var}\left(u_{(3) j}\right)=\mathbf{\tau}^{2}{ }_{(3)}$ is the level-3 heterogeneity variance, and $\operatorname{Var}\left(e_{i j}\right)=v^{2}$ ij is the known sampling variance. Both ML and REML estimation methods can be used to fit this model.

We may extend the idea of $I^{2}$ to the three-level meta-analysis (Cheung, 2014a). Specifically, we may define the level-2 $I_{(2)}^{2}$ and level-3 $I_{(3)}^{2}$ as:

$$
\begin{aligned}
& I_{(2)}^{2}=\frac{\hat{\tau}_{(2)}^{2}}{\hat{\tau}_{(2)}^{2}+\hat{\tau}_{(3)}^{2}+\tilde{v}}, \\
& I_{(3)}^{2}=\frac{\hat{\tau}_{(3)}^{2}}{\hat{\tau}_{(2)}^{2}+\hat{\tau}_{(3)}^{2}+\tilde{v}},
\end{aligned}
$$

where $\tilde{v}$ is a typical within-study sampling variance, such as that discussed before. These two indices can be interpreted as the between-study variances that can be explained by the level-2 or level-3 clusters.

Based on the idea that studies are also variables (Mehta \& Neale, 2005), Cheung (2014a) proposed a structural equation model to fit a three-level meta-analysis. Let us consider an example with a maximum of two effect sizes per cluster. We may rearrange the data to a wide format so that each row presents one cluster and the effect sizes within a cluster are treated as variables. In other words, if the maximum number of effect sizes per 
cluster is 5 , we will create 5 variables to store the effect sizes. In studies with fewer effect sizes, these "missing” effect sizes are treated as missing values.

Figure 8 displays a three-level meta-analysis with a maximum of two effect sizes per cluster. The level-3 population effect sizes are represented by a latent variable $f_{j} \sim N\left(\beta_{0}, \tau^{2}{ }_{(3)}\right)$, whereas the level-2 random-effect is represented by $u_{(2) i j} \sim N\left(0, \tau^{2}{ }_{(2)}\right)$. Since these "variables" are exchangeable, appropriate equality constraints are required. In general, the model-implied means and the variance-covariance matrix with $p$ variables in the $j$ th cluster are

$$
\begin{gathered}
\boldsymbol{\mu}_{j \times 1}(\boldsymbol{\theta})=\underset{p \times 1}{\mathbf{1}} \beta_{0}, \\
\underset{p \times p}{\boldsymbol{\Sigma}_{j}(\boldsymbol{\theta})}=\underset{p \times p}{\mathbf{1}} \tau_{(3)}^{2}+\underset{p \times p}{\boldsymbol{I}} \tau_{(2)}^{2}+\underset{p \times p}{\boldsymbol{V}_{j},}
\end{gathered}
$$

where $\mathbf{1}$ is a vector or matrix of ones, $\boldsymbol{I}$ is an identity matrix with the appropriate dimensions, and $\boldsymbol{V}_{j}$ is a diagonal matrix of the known sampling variances in the $j$ th cluster. Both ML and REML estimation methods can be implemented to fit this model in SEM. Moreover, level-2 and level-3 covariates can be added to explain the heterogeneity of variances and to calculate level-2 and level-3 R²s using Equation (16) (Cheung, 2014a; Cheung, 2015a, Chapter 6).

Before moving to the next section, we have to discuss and compare the similarities and differences between the multivariate and three-level meta-analyses. Both techniques are used to handle non-independent effect sizes in a meta-analysis. However, the causes of nonindependence are different (Cheung, 2019b). In a multivariate meta-analysis, the sampling errors of the effect sizes are correlated because the same participants are used in calculating the effect sizes. On the other hand, the non-independence in a three-level meta-analysis is due to the nested structure of the effect sizes although there is no overlap in participants when calculating the effect sizes. By imposing suitable constraints, Cheung (2015a, Chapter 6) showed the three-level meta-analysis is a special case of the multivariate meta-analysis. Similarly, a multivariate meta-analysis can be approximately analyzed within a three-level 
model by assuming a few assumptions. An interesting consequence of the latter observation is that some researchers (e.g., Van den Noortgate et al., 2013) suggested using a three-level model to analyze multivariate effect sizes when the sampling covariances are unavailable. Strictly speaking, this is not correct because the types of non-independence are different under these two models. Empirical simulations (e.g., Moeyaert et al., 2017), however, suggest that the approach works reasonably well under the studied conditions.

\section{Illustrations with the SEM-Based Meta-Analysis}

This section illustrates how to use the SEM approach to fit meta-analytic models using the metaSEM package (Cheung, 2015b). The metaSEM package has functions to facilitate the model fitting of the univariate and multivariate meta-analyses with the "meta" function and the three-level meta-analysis with the "meta3" function. For didactic purposes, we specify the models using the lavaan syntax (Rosseel, 2012), convert the models to RAM specifications (McArdle \& McDonald, 1984), and fit them using the OpenMx package (Neale et al., 2016). These datasets were also used in Cheung (2019b), which included the Mplus code (Muthén \& Muthén, 2017) for the same analyses. All of the data and the computer code are available at https://github.com/mikewlcheung/code-in-articles.

\section{Univariate Meta-Analysis}

The first example was adopted from Stadler et al. (2015, Table 1), who investigated the association between complex problem solving and intelligence by examining 60 independent samples from 47 studies. The authors conducted a meta-analysis without taking the dependence of the effect sizes into account. Based on the information on "Authors" and "Year," published in their Table 1, we identified 44 clusters. As an illustration, we first conducted the meta-analysis ignoring the cluster effect. A three-level meta-analysis is presented in the next section. 
When we fitted a fixed-effect model, the estimated common correlation (and its $S E$ ) was .65 (.00), $p<.01$. However, the $Q$ statistic was $\chi^{2}(d f=59)=1,155.14, p<.01$, suggesting that the assumption of the homogeneity of effect sizes was questionable. We fitted a randomeffects model. The estimated average effect (and its $S E$ ) was .42 (.03), $p<.01$; and its heterogeneity variance (and $I^{2}$ ) was .04 (.97). The results suggest that the random-effects model fits the data better. We conducted a mixed-effects meta-analysis using a binary study characteristic of GI (general intelligence represented by 1 vs. reasoning represented by 0 ) to explore the heterogeneity of the data. The regression slope (and its $S E$ ) was -.13 (.06), $p=.03$, $R^{2}=.11$. The findings show that studies using general intelligence are weaker than those using reasoning.

\section{Three-level Meta-Analysis}

The above results ignored the fact that the effect sizes were nested within studies. The number of effect sizes per study varied from 1 to 4, although most studies only contributed one effect size. We reran the analyses by taking the nested effect under the three-level metaanalysis. The estimated average correlation (and its $S E$ ) was .43 (.03), $p<.01$. The estimated level-2 and level-3 heterogeneity variances (and their $I^{2}$ ) were $.02(.45)$ and $.02(.51)$, respectively. When we compared the findings between the univariate meta-analysis ignoring the cluster effect and the three-level meta-analysis, we found that the estimated fixed effects were similar. By contrast, the heterogeneity variance in the univariate meta-analysis was almost the sum of the level-2 and level-3 heterogeneity variances.

We further included general intelligence as a covariate in the mixed-effects metaanalysis. The estimated regression slope (and its $S E$ ) was -.13 (.06), $p=.03$. The level-2 and level-3 $R^{2}$ were .08 and .13 , respectively. The findings suggest that the correlation is weaker when general intelligence rather than reasoning is used as the measure. 


\section{Multivariate Meta-Analysis}

The second example was taken from Nam et al. (2003, Table 1). These authors illustrated a multivariate meta-analysis when studying the effects of environmental tobacco smoke or passive smoking on the health of children. The effect sizes were the log-odds ratios of the group with environmental tobacco smoke against a normal control group in the development of asthma and lower respiratory disease. Since the correlation between asthma and lower respiratory disease was not available in the paper, we used a correlation of 0.5 to calculate the sampling covariance between the effect sizes. There were 59 studies in the data set.

We first fitted a fixed-effects model. The estimated common effects (and their SEs) on asthma and lower respiratory disease were .09 (.02), $p<.01$, and .08 (.01), $p<.01$, respectively. The $Q$ statistic in testing the homogeneity of effect sizes was $\chi^{2}(d f=65)=282.73, p<.01$. The results indicated that the fixed-effects model might not be appropriate. We then fitted a random-effects model. The estimated average effects (and their SEs) on asthma and lower respiratory disease were $.27(.05), p<.01$ and $.31(.05), p<.01$, respectively. It should be noted that the estimates based on the fixed- and random-effects models were quite different in this example. The estimated heterogeneity variances on asthma and lower respiratory disease (and their $I^{2}$ ) were $0.07(.84)$ and $0.05(.95)$, respectively.

We included the mean age of the participants as a covariate in exploring the heterogeneity of the effect sizes. The estimated slopes (and their SEs) on asthma and lower respiratory disease were $-.04(.02), p<.05$, and $-.02(.01), p<.05$, respectively. The estimated $R^{2} \mathrm{~s}$ on asthma and lower respiratory disease were .59 and .39 , respectively. The findings show that the risk of developing asthma and lower respiratory disease was found to be greater in studies with younger participants. It is of importance to note that the mean age of the 
participants, not the age of the participants, was used in the meta-analysis. We need to be mindful of avoiding ecological fallacies when interpreting the results.

Although we did not illustrate this in the present analyses, the SEM approach makes it easy to test constraints, such as (1) whether the average effects on asthma and lower respiratory disease are the same, (2) whether the heterogeneity variances on asthma and lower respiratory disease are the same, and (3) whether the effects of the mean age of the participants on asthma and lower respiratory disease are the same.

\section{Discussion and Future Directions}

The main goal of this chapter was to introduce the SEM-based meta-analysis, in which meta-analytic models are fitted as structural equation models. We showed how fixedeffect, random-effects, and mixed-effects meta-analyses could be integrated into the SEM framework. Using SEM, we also demonstrated how easily we could extend these models to more complex models, including multivariate and three-level meta-analyses.

An extension of multivariate meta-analysis that was not elaborated upon in this chapter is meta-analytic structural equation modeling (MASEM; Cheung, 2014b; 2021; Cheung \& Chan, 2005; 2009; Cheung \& Cheung, 2016; Jak \& Cheung, 2018; 2020). It combines correlation matrices and fits structural equation models on the average correlation matrix. In the first stage of the analysis in MASEM, an average correlation matrix is obtained using the random-effects model. In the second stage of the analysis, a correlation structure is fitted on the average correlation matrix with the WLS estimation method by using the inverse of the sampling covariance matrix of the average correlation matrix as the weight matrix.

Another extension of multivariate meta-analysis is network meta-analysis (Dias et al., 2018), in which multiple treatments are compared simultaneously in a single analysis by combining direct and indirect evidence within a network of randomized controlled trials. Tu 
and his colleagues (Shih \& Tu, 2019; Tu \& Wu, 2017) extended the SEM-based metaanalysis to analyze network meta-analysis by introducing the appropriate constraints.

The combination of meta-analysis and SEM also raises several avenues for research on the limitations of the current SEM framework and software. First, only a few SEM packages (OpenMx and Mplus) are capable of analyzing meta-analytic data via definition variables. Perhaps one main reason for this is that most SEM users are mainly interested in analyzing subject-level data, for which the assumption is that the data are independent and identically distributed. The functionalities of the current SEM packages will be greatly expanded if known sampling variances and covariances can easily be incorporated into the models. The second issue is that it is mainly ML estimation that is used in SEM. There are other estimation methods, such as the unweighted least squares and generalized least squares methods. How these estimators work in meta-analysis data has yet to be studied. On the other hand, there are many estimators in a meta-analysis that are not available in conventional SEM packages (Viechtbauer, 2010). It is of interest to see how these estimators can be implemented in standard SEM packages.

Third, the use of raw data in a meta-analysis is called individual patient data in medicine (Higgins et al., 2001) or integrative data analysis in psychology (Hussong et al., 2013). It is essentially a multilevel model on the raw data. SEM fits nicely as a modeling framework to analyze the data. It would be a positive trend if more such applications were to use SEM as the framework of analysis. To summarize, many structural equation models can be extended to meta-analytic data by taking the known sampling variances and covariances into account. We hope that the integration of meta-analysis and SEM will benefit researchers in both meta-analysis and SEM. 


\section{References}

Aiken, L. S., West, S. G., \& Millsap, R. E. (2008). Doctoral training in statistics, measurement, and methodology in psychology: Replication and extension of Aiken, West, Sechrest, and Reno’s (1990) Survey of PhD programs in North America. American Psychologist, 63(1), 32-50. https://doi.org/10.1037/0003-066X.63.1.32

Bauer, D. J. (2003). Estimating multilevel linear models as structural equation models. Journal of Educational and Behavioral Statistics, 28(2), 135-167. https://doi.org/10.3102/10769986028002135

Bentler, P. M. (1986). Structural modeling and psychometrika: An historical perspective on growth and achievements. Psychometrika, 51(1), 35-51. https://doi.org/10.1007/BF02293997

Bentler, P. M. (2006). EQS 6 structural equations program manual. Multivariate Software. Bollen, K. A. (1989). Structural equations with latent variables. Wiley.

Bonett, D. G. (2008). Meta-analytic interval estimation for bivariate correlations. Psychological Methods, 13(3), 173-181. https://doi.org/10.1037/a0012868

Borenstein, M., Hedges, L. V., Higgins, J. P. T., \& Rothstein, H. R. (2009). Introduction to meta-analysis. John Wiley \& Sons.

Cheung, M. W.-L. (2008). A model for integrating fixed-, random-, and mixed-effects metaanalyses into structural equation modeling. Psychological Methods, 13(3), 182-202. https://doi.org/10.1037/a0013163

Cheung, M. W.-L. (2010). Fixed-effects meta-analyses as multiple-group structural equation models. Structural Equation Modeling: A Multidisciplinary Journal, 17(3), 481-509. https://doi.org/10.1080/10705511.2010.489367

Cheung, M. W.-L. (2013a). Implementing restricted maximum likelihood estimation in 
structural equation models. Structural Equation Modeling: A Multidisciplinary Journal, 20(1), 157-167. https://doi.org/10.1080/10705511.2013.742404

Cheung, M. W.-L. (2013b). Multivariate meta-analysis as structural equation models. Structural Equation Modeling: A Multidisciplinary Journal, 20(3), 429-454. https://doi.org/10.1080/10705511.2013.797827

Cheung, M. W.-L. (2014a). Modeling dependent effect sizes with three-level meta-analyses: A structural equation modeling approach. Psychological Methods, 19(2), 211-229. https://doi.org/10.1037/a0032968

Cheung, M. W.-L. (2014b). Fixed- and random-effects meta-analytic structural equation modeling: Examples and analyses in R. Behavior Research Methods, 46(1), 29-40. https://doi.org/10.3758/s13428-013-0361-y

Cheung, M. W.-L. (2015a). Meta-analysis: A structural equation modeling approach. John Wiley \& Sons, Inc. http://dx.doi.org/10.1002/9781118957813

Cheung, M. W.-L. (2015b). metaSEM: An R package for meta-analysis using structural equation modeling. Frontiers in Psychology, 5(1521). https://doi.org/10.3389/fpsyg.2014.01521

Cheung, M. W.-L. (2018). Computing multivariate effect sizes and their sampling covariance matrices with structural equation modeling: Theory, examples, and computer simulations. Frontiers in Psychology, 9(1387). https://doi.org/10.3389/fpsyg.2018.01387

Cheung, M. W.-L. (2019a). Some reflections on combining meta-analysis and structural equation modeling. Research Synthesis Methods, 10(1), 15-22. https://doi.org/10.1002/jrsm.1321

Cheung, M. W.-L. (2019b). A guide to conducting a meta-analysis with non-independent 
effect sizes. Neuropsychology Review, 29(4), 387-396.

https://doi.org/10.1007/s11065-019-09415-6

Cheung, M. W.-L. (2021). Meta-analytic structural equation modeling. In Oxford Research Encyclopedia of Business and Management. Oxford University Press. https://doi.org/10.1093/acrefore/9780190224851.013.225

Cheung, M. W.-L., \& Chan, W. (2005). Meta-analytic structural equation modeling: A twostage approach. Psychological Methods, 10(1), 40-64. https://doi.org/10.1037/1082989X.10.1.40

Cheung, M. W.-L., \& Chan, W. (2009). A two-stage approach to synthesizing covariance matrices in meta-analytic structural equation modeling. Structural Equation Modeling: A Multidisciplinary Journal, 16(1), 28-53.

https://doi.org/10.1080/10705510802561295

Cheung, M. W.-L., \& Cheung, S. F. (2016). Random-effects models for meta-analytic structural equation modeling: Review, issues, and illustrations. Research Synthesis Methods, 7(2), 140-155. https://doi.org/10.1002/jrsm.1166

Cochran, W. (1954). The combination of estimates from different experiments. Biometrics, 10(1), 101-129.

Cooper, H. M. (2017). Research synthesis and meta-analysis: A step-by-step approach (5th ed.). Sage Publications, Inc.

Curran, P. J. (2003). Have multilevel models been structural equation models all along? Multivariate Behavioral Research, 38(4), 529-569. https://doi.org/10.1207/s15327906mbr3804_5

DerSimonian, R., \& Laird, N. (1986). Meta-analysis in clinical trials. Controlled Clinical Trials, 7(3), 177-188. https://doi.org/10.1016/0197-2456(86)90046-2 
Dias, S., Ades, A. E., Welton, N. J., Jansen, J. P., \& Sutton, A. J. (2018). Network metaanalysis for decision making. John Wiley \& Sons, Ltd. https://doi.org/10.1002/9781118951651.ch1

Dimou, N. L., Adam, M., \& Bagos, P. G. (2016). A multivariate method for meta-analysis and comparison of diagnostic tests. Statistics in Medicine, n/a-n/a. https://doi.org/10.1002/sim.6919

Enders, C. K. (2010). Applied missing data analysis. Guilford Press.

Fischer, R., \& Boer, D. (2011). What is more important for national well-being: Money or autonomy? A meta-analysis of well-being, burnout, and anxiety across 63 societies. Journal of Personality and Social Psychology, 101(1), 164-184. https://doi.org/10.1037/a0023663

Glass, G. V. (1976). Primary, secondary, and meta-analysis of research. Educational Researcher, 5(10), 3-8. https://doi.org/10.2307/1174772

Glockner-Rist, A., \& Hoijtink, H. (2003). The best of both worlds: Factor analysis of dichotomous data using item response theory and structural equation modeling. Structural Equation Modeling: A Multidisciplinary Journal, 10(4), 544-565. https://doi.org/10.1207/S15328007SEM1004_4

Hedges, L. V. (1981). Distribution theory for Glass's estimator of effect size and related estimators. Journal of Educational and Behavioral Statistics, 6(2), 107-128. https://doi.org/10.3102/10769986006002107

Hedges, L. V. (1983). A random effects model for effect sizes. Psychological Bulletin, 93(2), 388-395. https://doi.org/10.1037/0033-2909.93.2.388

Hedges, L. V. (2007). Meta-analysis. In C. R. Rao \& S. Sinharay (Eds.), Psychometrics (Vol. 26, pp. 919-953). Russell Sage Foundation. 
Hedges, L. V., \& Olkin, I. (1985). Statistical methods for meta-analysis. Academic Press. Hedges, L. V., \& Vevea, J. L. (1998). Fixed- and random-effects models in meta-analysis. Psychological Methods, 3(4), 486-504. https://doi.org/10.1037/1082-989X.3.4.486

Higgins, J. P. T., \& Thompson, S. G. (2002). Quantifying heterogeneity in a meta-analysis. Statistics in Medicine, 21(11), 1539-1558. https://doi.org/10.1002/sim.1186

Higgins, J. P. T., Thompson, S. G., Deeks, J. J., \& Altman, D. G. (2003). Measuring inconsistency in meta-analyses. British Medical Journal, 327(7414), 557-560.

Higgins, J., Whitehead, A., Turner, R., Omar, R., \& Thompson, S. (2001). Meta-analysis of continuous outcome data from individual patients. Statistics in Medicine, 20(15), 2219-2241.

Hussong, A. M., Curran, P. J., \& Bauer, D. J. (2013). Integrative data analysis in clinical psychology research. Annual Review of Clinical Psychology, 9(1), 61-89. https://doi.org/10.1146/annurev-clinpsy-050212-185522

Jackson, D., Riley, R., \& White, I. R. (2011). Multivariate meta-analysis: Potential and promise. Statistics in Medicine, 30(20), 2481-2498. https://doi.org/10.1002/sim.4172 Jackson, D., White, I. R., \& Riley, R. D. (2012). Quantifying the impact of between-study heterogeneity in multivariate meta-analyses. Statistics in Medicine, 31(29), 38053820. https://doi.org/10.1002/sim.5453

Jackson, D., White, I. R., \& Riley, R. D. (2013). A matrix-based method of moments for fitting the multivariate random effects model for meta-analysis and meta-regression. Biometrical Journal, 55(2), 231-245. https://doi.org/10.1002/bimj.201200152

Jak, S., \& Cheung, M. W.-L. (2018). Testing moderator hypotheses in meta-analytic structural equation modeling using subgroup analysis. Behavior Research Methods, 50(4), 1359-1373. https://doi.org/10.3758/s13428-018-1046-3 
Jak, S., \& Cheung, M. W.-L. (2020). Meta-analytic structural equation modeling with moderating effects on SEM parameters. Psychological Methods, 25(4), 430-455. https://doi.org/10.1037/met0000245

Jöreskog, K. G. (1967). Some contributions to maximum likelihood factor analysis. Psychometrika, 32(4), 443-482. https://doi.org/10.1007/BF02289658

Jöreskog, K. G., \& Sörbom, D. (1996). LISREL 8: A user's reference guide. Scientific Software International, Inc.

Kalaian, H. A., \& Raudenbush, S. W. (1996). A multivariate mixed linear model for metaanalysis. Psychological Methods, 1(3), 227-235. https://doi.org/10.1037/1082989X.1.3.227

Kelley, K., \& Preacher, K. J. (2012). On effect size. Psychological Methods, 17(2), 137-152. https://doi.org/10.1037/a0028086

Kline, R. B. (2016). Principles and practice of structural equation modeling (4th ed.). Guilford Press.

Konstantopoulos, S. (2011). Fixed effects and variance components estimation in three-level meta-analysis. Research Synthesis Methods, 2(1), 61-76. https://doi.org/10.1002/jrsm.35

Lubke, G., \& Muthén, B. O. (2007). Performance of factor mixture models as a function of model size, covariate effects, and class-specific parameters. Structural Equation Modeling: A Multidisciplinary Journal, 14(1), 26-47. https://doi.org/10.1080/10705510709336735

McArdle, J. J., \& McDonald, R. P. (1984). Some algebraic properties of the Reticular Action Model for moment structures. British Journal of Mathematical and Statistical Psychology, 37(2), 234-251. https://doi.org/10.1111/j.2044-8317.1984.tb00802.x 
Mehta, P. D., \& Neale, M. C. (2005). People are variables too: Multilevel structural equations modeling. Psychological Methods, 10(3), 259-284. https://doi.org/10.1037/1082989X.10.3.259

Moeyaert, M., Ugille, M., Beretvas, S. N., Ferron, J., Bunuan, R., \& Noortgate, W. V. den. (2017). Methods for dealing with multiple outcomes in meta-analysis: A comparison between averaging effect sizes, robust variance estimation and multilevel metaanalysis. International Journal of Social Research Methodology, 20(6), 559-572. https://doi.org/10.1080/13645579.2016.1252189

Muthén, B. (1984). A general structural equation model with dichotomous, ordered categorical, and continuous latent variable indicators. Psychometrika, 49(1), 115-132. https://doi.org/10.1007/BF02294210

Muthén, B. O. (1994). Multilevel covariance structure analysis. Sociological Methods \& Research, 22(3), 376-398. https://doi.org/10.1177/0049124194022003006

Muthén, B. O., \& Muthén, L. K. (2017). Mplus user’s guide (8th ed.). Muthén \& Muthén.

Nam, I.-S., Mengersen, K., \& Garthwaite, P. (2003). Multivariate meta-analysis. Statistics in Medicine, 22(14), 2309-2333. https://doi.org/10.1002/sim.1410

Neale, M. C., Hunter, M. D., Pritikin, J. N., Zahery, M., Brick, T. R., Kirkpatrick, R. M., Estabrook, R., Bates, T. C., Maes, H. H., \& Boker, S. M. (2016). OpenMx 2.0: Extended structural equation and statistical modeling. Psychometrika, 81(2), 535-549. https://doi.org/10.1007/s11336-014-9435-8

R Development Core Team. (2021). R: a language and environment for statistical computing. http://www.R-project.org/

Raudenbush, S. W. (2009). Analyzing effect sizes: Random effects models. In H. M. Cooper, L. V. Hedges, \& J. C. Valentine (Eds.), The handbook of research synthesis and meta- 
analysis (2nd ed., pp. 295-315). Russell Sage Foundation.

Raudenbush, S. W., Becker, B. J., \& Kalaian, H. (1988). Modeling multivariate effect sizes. Psychological Bulletin, 103(1), 111-120. https://doi.org/10.1037/0033-2909.103.1.111

Raykov, T., \& Marcoulides, G. A. (2004). Using the Delta method for approximate interval estimation of parameter functions in SEM. Structural Equation Modeling: A Multidisciplinary Journal, 11(4), 621-637. https://doi.org/10.1207/s15328007sem1104_7

Rindskopf, D. (1984). Using phantom and imaginary latent-variables to parameterize constraints in linear structural models. Psychometrika, 49(1), 37-47.

Rosseel, Y. (2012). lavaan: An R package for structural equation modeling. Journal of Statistical Software, 48(2), 1-36.

Rovine, M. J., \& Molenaar, P. C. M. (2000). A structural modeling approach to a multilevel random coefficients model. Multivariate Behavioral Research, 35(1), 51-88. https://doi.org/10.1207/S15327906MBR3501_3

Schmidt, F. L., \& Hunter, J. E. (2015). Methods of meta-analysis: Correcting error and bias in research findings (3rd ed.). Sage.

Schoemann, A. M. (2016). Using multiple group modeling to test moderators in metaanalysis. Research Synthesis Methods, 7(4), 387-401. https://doi.org/10.1002/jrsm.1200

Shih, M.-C., \& Tu, Y.-K. (2019). Evaluating network meta-analysis and inconsistency using arm-parameterized model in structural equation modeling. Research Synthesis Methods, 10(2), 240-254. https://doi.org/10.1002/jrsm.1344

Stadler, M., Becker, N., Gödker, M., Leutner, D., \& Greiff, S. (2015). Complex problem solving and intelligence: A meta-analysis. Intelligence, 53, 92-101. 
https://doi.org/10.1016/j.intell.2015.09.005

Stanley, T. D., \& Doucouliagos, H. (2015). Neither fixed nor random: Weighted least squares meta-analysis. Statistics in Medicine, 34(13), 2116-2127. https://doi.org/10.1002/sim.6481

Takane, Y., \& Deleeuw, J. (1987). On the relationship between item response theory and factor-analysis of discretized variables. Psychometrika, 52(3), 393-408. https://doi.org/10.1007/BF02294363

Takkouche, B., Cadarso-Suárez, C., \& Spiegelman, D. (1999). Evaluation of old and new tests of heterogeneity in epidemiologic meta-analysis. American Journal of Epidemiology, 150(2), 206-215.

Tu, Y.-K., \& Wu, Y.-C. (2017). Using structural equation modeling for network metaanalysis. BMC Medical Research Methodology, 17(104). https://doi.org/10.1186/s12874-017-0390-9

Van den Noortgate, W., López-López, J. A., Marín-Martínez, F., \& Sánchez-Meca, J. (2013). Three-level meta-analysis of dependent effect sizes. Behavior Research Methods, 45(2), 576-594. https://doi.org/10.3758/s13428-012-0261-6

van Houwelingen, H. C., Arends, L. R., \& Stijnen, T. (2002). Advanced methods in metaanalysis: Multivariate approach and meta-regression. Statistics in Medicine, 21(4), 589-624. https://doi.org/10.1002/sim.1040

Viechtbauer, W. (2005). Bias and efficiency of meta-analytic variance estimators in the random-effects model. Journal of Educational and Behavioral Statistics, 30(3), 261293. https://doi.org/10.3102/10769986030003261

Viechtbauer, W. (2010). Conducting meta-analyses in R with the metafor package. Journal of Statistical Software, 36(3), 1-48. https://doi.org/10.18637/jss.v036.i03 
Xiong, C., Miller, J. P., \& Morris, J. C. (2010). Measuring study-specific heterogeneity in meta-analysis: Application to an antecedent biomarker study of alzheimer's disease. Statistics in Biopharmaceutical Research, 2(3), 300-309. https://doi.org/10.1198/sbr.2009.0067

Yuan, K.-H., Yang-Wallentin, F., \& Bentler, P. M. (2012). ML versus MI for missing data with violation of distribution conditions. Sociological Methods \& Research, 41(4), 598-629. https://doi.org/10.1177/0049124112460373 
Table 1: Concepts between Meta-Analysis and SEM

SEM

Meta-Analysis

Subject ( $N$ subjects)

Study ( $k$ studies)

Indicator or observed variable

Observed effect size

Latent variable

True or population effect size

Mean of the latent variable

Average effect

Variance of the latent variable

Heterogeneity variance

Known variance of measurement error in the Known sampling variance in the $i$ th study

ith subject

Predictor or covariate

Study characteristic, predictor, covariate, or

moderator 
Table 2: Common Effect Sizes and Their Sampling Variances

$\begin{array}{cccc}\begin{array}{c}\text { Quantity of } \\ \text { interest }\end{array} & \begin{array}{c}\text { Summary statistics required to } \\ \text { compute the effect sizes }\end{array} & \text { Effect size }\left(y_{i}\right) & \text { Approximate sampling variance } \\ \left(v_{i}\right)\end{array}$

$\begin{array}{lll}\text { Relative risk }(R R) & a \text { : frequency of success in Group } 1 & \\ \text { Odds ratio }(O R) & b \text { : frequency of failure in Group } 1 & y_{R R}=\log \left(\frac{a * n_{2}}{c * n_{1}}\right)\end{array}$
$n_{1}=a+b$
$c$ : frequency of success in Group $2 y_{O R}=\log \left(\frac{a * d}{b * c}\right)$
$d$ : frequency of failure in Group 2

$$
\begin{aligned}
& v_{R R}=\frac{1}{a}-\frac{1}{n_{1}}+\frac{1}{c}-\frac{1}{n_{2}} \\
& v_{O R}=\frac{1}{a}+\frac{1}{b}+\frac{1}{c}+\frac{1}{d}
\end{aligned}
$$

$n_{2}=c+d$

$$
\begin{array}{llll}
\hline \text { Raw mean } & \bar{X}_{1}: \text { Sample mean for Group 1 } & y_{R M D}=\bar{X}_{1}-\bar{X}_{2} & v_{R M D}=S_{\text {pooled }}^{2}\left(\frac{1}{n_{1}}+\frac{1}{n_{2}}\right) \\
\text { difference (RMD) } & S_{1}^{2}: \text { Sample variance for Group 1 } & \\
\text { Standardized } & n_{1}: \text { Sample size for Group 1 } & y_{S M D}=\left(1-\frac{3}{4\left(n_{1}+n_{2}\right)-9}\right) \frac{\bar{X}_{1}-\bar{X}_{2}}{S_{\text {pooled }}} & v_{S M D}=\frac{n_{1}+n_{2}}{n_{1} n_{2}}+\frac{y_{S M D}^{2}}{2\left(n_{1}+n_{2}\right)} \\
\text { mean difference } & \bar{X}_{2}: \text { Sample mean for Group 2 } & \\
\text { (SMD) } & S_{2}^{2}: \text { Sample variance for Group 2 } & \\
& n_{2}: \text { Sample size for Group 2 } \\
& S_{\text {pooled }}^{2}=\frac{\left(n_{1}-1\right) S_{1}^{2}+\left(n_{2}-1\right) S_{2}^{2}}{n_{1}+n_{2}-2}
\end{array}
$$

$\bar{X}_{1}:$ Sample mean for Group $1 \quad y_{R M D}=\bar{X}_{1}-\bar{X}_{2}$

$r$ : sample correlation coefficient

$n$ : Sample size

\begin{tabular}{ll}
\hline Correlation $(\mathrm{r})$ & $r$ : sample corre \\
Fisher's $z$ & $n$ : Sample size
\end{tabular}

transformed score

$$
\begin{array}{ll}
y_{r}=r & v_{r}=\frac{\left(1-r^{2}\right)^{2}}{n-1} \\
y_{z}=0.5 * \log \left(\frac{1+r}{1-r}\right) & v_{z}=\frac{1}{n-3}
\end{array}
$$

Reprinted by permission from Springer Nature: Neuropsychology Review. "A Guide to Conducting a Meta-Analysis," by Mike W.-L. Cheung and Ranjith Vijayakumar, Copyright (2016). 
Figure 1. A fixed-effect meta-analysis.

Figure 2. A random-effects meta-analysis.

Figure 3. A mixed-effects meta-analysis treating the covariate as a variable.

Figure 4. A mixed-effects meta-analysis allowing missing covariate.

Figure 5. A mixed-effects meta-analysis treating the covariate as a design matrix.

Figure 6. A multivariate random-effects meta-analysis.

Figure 7. A multivariate mixed-effects meta-analysis with one covariate.

Figure 8. A three-level meta-analysis with two effect sizes per cluster. 
$v_{i}$

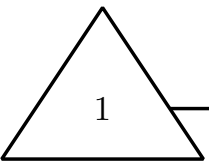

$\beta_{\mathrm{F}}$

Q

$y_{i}$ 


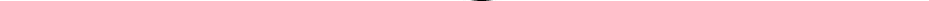




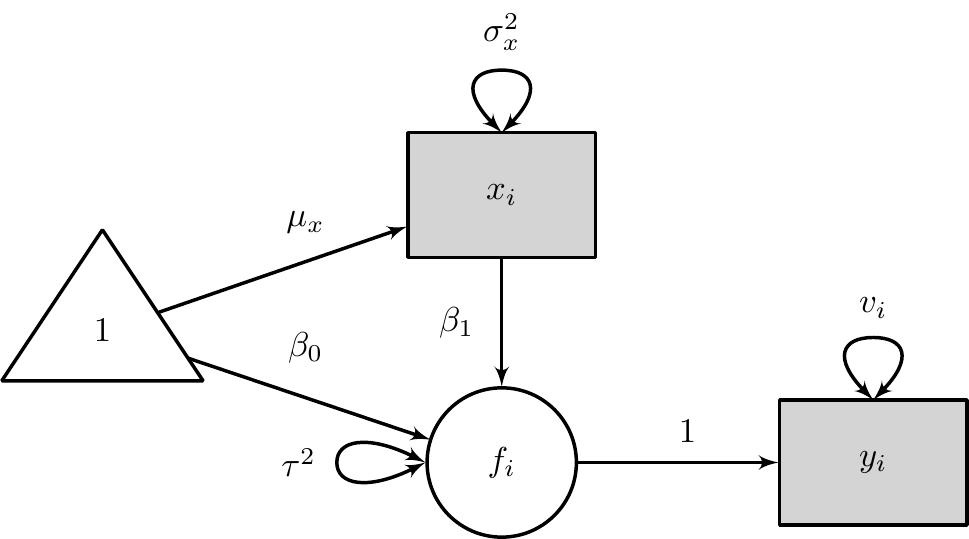




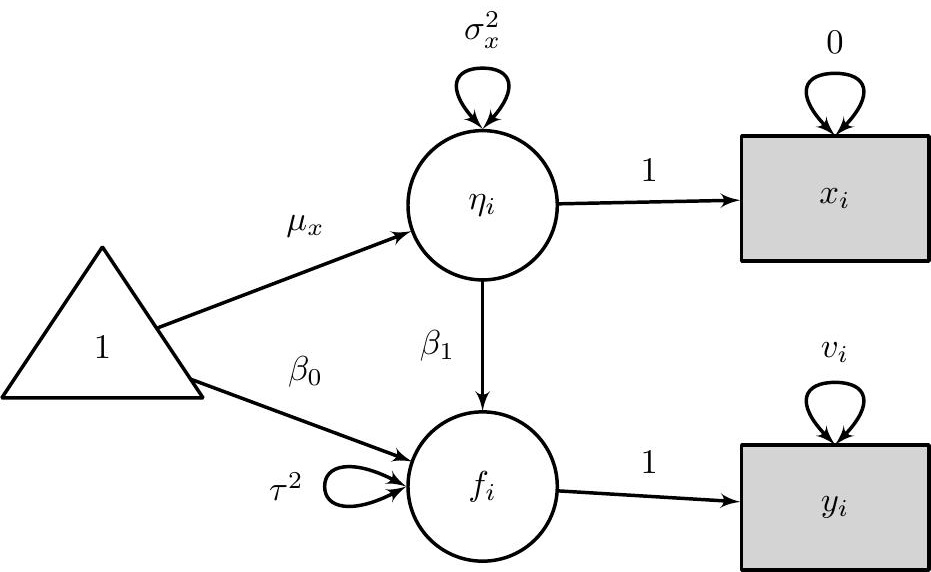




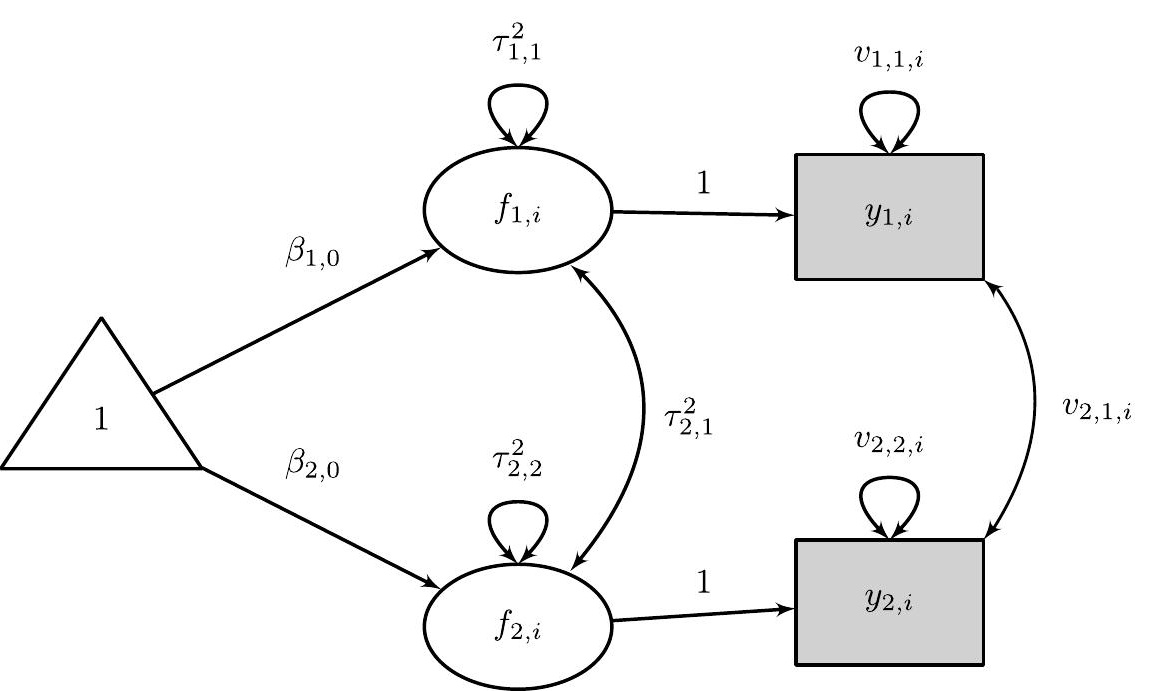




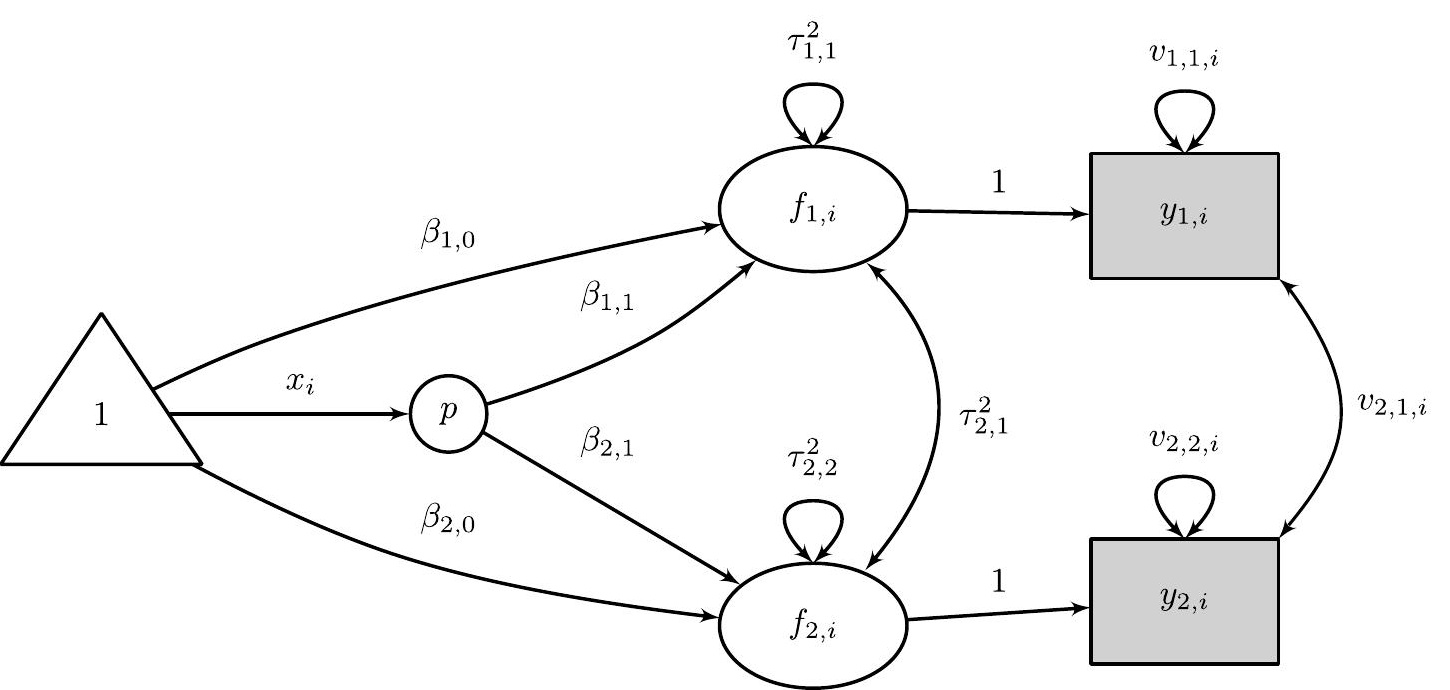




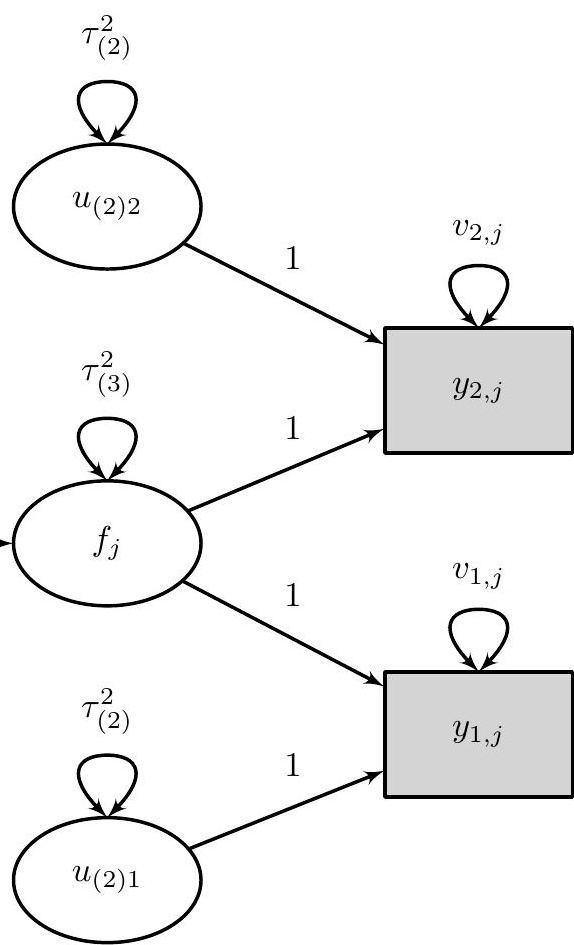

